\title{
The unidentified TeV source (TeV J2032+4130) and surrounding field: Final HEGRA IACT-System results
}

\author{
F. Aharonian ${ }^{1}$, A. Akhperjanian ${ }^{7}$, M. Beilicke ${ }^{4}$, K. Bernlöhrr ${ }^{1}$, H.-G. Börst ${ }^{5}$, H. Bojahr $^{6}$, O. Bolz ${ }^{1}$, T. Coarasa ${ }^{2}$, \\ J. Contreras ${ }^{3}$, J. Cortina ${ }^{10}$, S. Denninghoff ${ }^{2}$, V. Fonseca ${ }^{3}$, M. Girma ${ }^{1}$, N. Götting ${ }^{4}$, G. Heinzelmann ${ }^{4}$, G. Hermann ${ }^{1}$, \\ A. Heusler ${ }^{1}$, W. Hofmann ${ }^{1}$, D. Horns ${ }^{1}$, I. Jung ${ }^{1,9}$, R. Kankanyan ${ }^{1}$, M. Kestel ${ }^{2}$, A. Kohnle ${ }^{1}$, A. Konopelko ${ }^{1,14}$, \\ D. Kranich ${ }^{2}$, H. Lampeitl ${ }^{4}$, M. Lopez ${ }^{3}$, E. Lorenz ${ }^{2}$, F. Lucarelli ${ }^{3}$, O. Mang 5 , D. Mazin ${ }^{2}$, H. Meyer $^{6}$, R. Mirzoyan ${ }^{2}$, \\ A. Moralejo ${ }^{3}$, E. Oña-Wilhelmi ${ }^{3}$, M. Panter ${ }^{1}$, A. Plyasheshnikov ${ }^{1,8}$, G. Pühlhofer ${ }^{11}$, R. de los Reyes ${ }^{3}$, W. Rhode ${ }^{6}$, \\ J. Ripken ${ }^{4}$, G. P. Rowell ${ }^{1}$, V. Sahakian ${ }^{7}$, M. Samorski ${ }^{5}$, M. Schilling ${ }^{5}$, M. Siems ${ }^{5}$, D. Sobzynska ${ }^{2,12}$, W. Stamm ${ }^{5}$, \\ M. Tluczykont ${ }^{4,13}$, V. Vitale ${ }^{2}$, H. J. Völk ${ }^{1}$, C. A. Wiedner ${ }^{1}$, and W. Wittek ${ }^{2}$
}

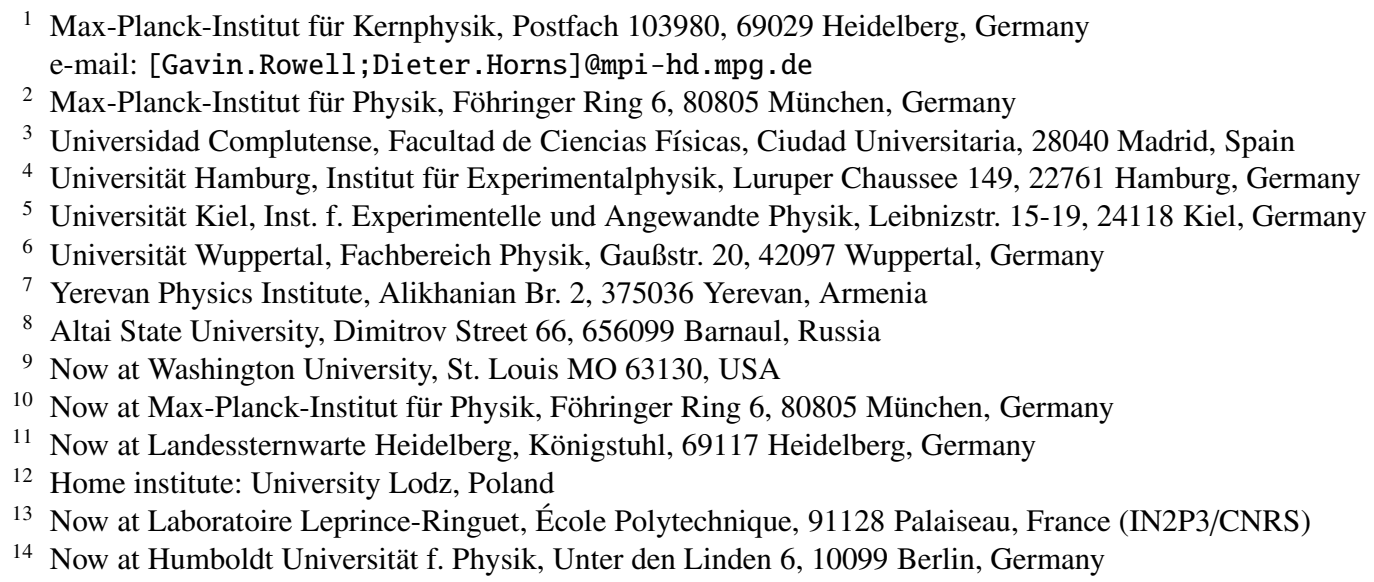

Received 29 June 2004 / Accepted 17 September 2004

\begin{abstract}
The unidentified $\mathrm{TeV}$ source in Cygnus is now confirmed by follow-up observations from 2002 with the HEGRA stereoscopic system of Cherenkov Telescopes. Using all data (1999 to 2002) we confirm this new source as steady in flux over the four years of data taking, extended with radius $6.2^{\prime}\left( \pm 1.2_{\text {stat }}^{\prime} \pm 0.9_{\text {sys }}^{\prime}\right)$ and exhibiting a hard spectrum with photon index -1.9. It is located in the direction of the dense OB stellar association, Cygnus OB2. Its integral flux above energies $E>1 \mathrm{TeV}$ amounts to $\sim 5 \%$ of the Crab assuming a Gaussian profile for the intrinsic source morphology. There is no obvious counterpart at radio, optical nor X-ray energies, leaving TeV J2032+4130 presently unidentified. Observational parameters of this source are updated here and some astrophysical discussion is provided. Also included are upper limits for a number of other interesting sources in the FoV, including the famous microquasar Cygnus X-3.
\end{abstract}

Key words. gamma rays: observations - stars: early-type - open clusters and associations: individual: Cygnus OB2

\section{Introduction}

The reasonably large fields of view (FoV, e.g. $F W H M \geq 3^{\circ}$ ) achieved by ground-based $\gamma$-ray telescopes permits surveytype observations using a few or even singly pointed observations. Such potential was realised with the serendipitous discovery of a $\mathrm{TeV}$ source in the Cygnus region. Analysis of archival data ( $\sim 121 \mathrm{~h}$ from 1999 to 2001) of the HEGRA system of Imaging Atmospheric Cherenkov Telescopes (HEGRA IACT-System) revealed convincing evidence for an apparently steady, spectrally hard ( -1.9 differential photon index) and possibly extended $\mathrm{TeV}$ source (Aharonian et al. 2002). Follow-up observations using the HEGRA IACT-System were performed during its final season of operation (2002) for a total of $\sim 158 \mathrm{~h}$. Analysis of these data again reveal the presence of this source, thus confirming its existence with the HEGRA IACT-System. Earlier, observations (1991) with the HEGRA scintillator array (Merck 1993) revealed a multi-TeV excess, positionally consistent with Cygnus X-3. Analysis with improved direction reconstruction (Krawczynski 1995) revealed this excess $(+4.3 \sigma$ pre-trial $)$ as centered roughly $0.5^{\circ}$ north of Cygnus X-3. Interestingly, the Crimean group (using the 
Cherenkov imaging technique) reported a significant excess $\left(\sim+6.0 \sigma\right.$ pre-trial) $\sim 0.7^{\circ}$ north of Cygnus X-3 (Neshpor et al. 1995), and recently, the Whipple collaboration also reported an excess at the HEGRA position $(+3.3 \sigma)$ in their archival data of 1989/1990 (Lang et al. 2004).

We summarise here in some detail our analysis and numerical results for TeV J2032+4130, and give a brief astrophysical interpretation. Given the large FoV of observations, deep exposures were also obtained for a number of other interesting sources. Upper limits for these sources are given.

\section{Data analysis \& results}

The HEGRA IACT-System, de-commissioned in September 2002, consisted of five identical Cherenkov telescopes (each with $8.5 \mathrm{~m}^{2}$ mirror area) on the Canary Island of La Palma (2200 m a.s.1.). Employing the stereoscopic technique, this system achieved an angular resolution $<0.1^{\circ}$ and energy resolution $<15 \%$ for $\gamma$-rays on an event-by-event basis over the $0.5 \mathrm{TeV}$ to $>50 \mathrm{TeV}$ regime. Detailed technical descriptions of the HEGRA IACT-System and performance can be found in Pühlhofer et al. (2003).

Gamma-ray-like events are preferentially selected against those of the dominant isotropic background of cosmic-rays (CR). This is achieved with cuts on $\theta$, the angular distance between the reconstructed and assumed arrival directions, and also the mean-scaled-width $\bar{w}$ (Aharonian et al. 2000a), which is a measure of an image's conformity to a $\gamma$-ray-like shape. Event arrival directions are reconstructed using the algorithms described in Hofmann et al. (1999). We a priori selected algorithm " 3 " for final analysis, but also employed the other available algorithms to check consistency of the signal. So-called tight cuts were implemented, namely: $\theta<0.12^{\circ}, \bar{w}<1.1$, which are optimal for point-like sources in a background-dominated scenario. We also required a minimum $n_{\text {tel }} \geq 3$ telescope images per event for the $\theta$ and $\bar{w}$ calculation (Aharonian et al. 2002). An estimate of the CR background surviving cuts is made using both the template (see Rowell 2003) and displaced background models for consistency checks on the source excess. The displacement background model employs different regions in the FoV for background estimation using events from $\bar{w}<1.1$.

In Tables 1 and 2 we summarise details of the TeV source which includes the excess significance, the source extension $\sigma_{\text {src }}$, both of which are calculated at the excess centre of gravity $(\mathrm{CoG})$, and also the energy spectrum and flux. Some results are split according to data subsets, 1999 to 2001 (dataset §1), and 2002 (dataset §2).

The CoG and source extension were estimated by fitting a 2D Gaussian convolved with the system point spread function (PSF) to a histogram of $\gamma$-ray-like $(\bar{w}<1.1)$ events binned over a $1^{\circ} \times 1^{\circ} \mathrm{FoV}$. The PSF is estimated from Crab data, giving a value which agrees with Monto Carlo simulations of a point source (Aharonian et al. 2000b, 2004). A Gaussian profile suitably describes the excess morphology (discussed later). Here, we used higher quality events selected according to the estimated error in reconstructed direction $\epsilon$ (see e.g. Hofmann et al. 2000a). This cut on $\epsilon$ reduced somewhat the systematic
Table 1. Numerical summary for $\mathrm{TeV} \mathrm{J} 2032+4130$. a) Centre of Gravity (CoG) and extension $\sigma_{\text {src }}$ (std. dev. of a 2D Gaussian); b) event summary. The values $s$ and $b$ are event numbers for the $\gamma$-ray-like and background (from the Template and Displaced models, see text) respectively, and $s-\alpha b$ is the excess using a normalisation $\alpha$. $S$ denotes the excess significance using Eq. (17) of Li \& Ma (1983); c) integral events after spectral cuts.

(a) CoG \& Extension $\left(\epsilon \leq 0.12^{\circ}\right)$

\begin{tabular}{|c|c|c|}
\hline \multicolumn{3}{|c|}{ — All Data $(278.3 \mathrm{~h})$ - } \\
\hline RA $\alpha_{2000}:$ & $20^{\mathrm{hr}} 31^{\mathrm{m}} 57.0^{\mathrm{s}}$ & $\pm 6.2_{\text {stat }}^{\mathrm{s}} \pm 13.7_{\text {sys }}^{\mathrm{s}}$ \\
\hline $\operatorname{Dec} \delta_{2000}:$ & $41^{\circ} 29^{\prime} 56.8^{\prime \prime}$ & $\pm 1.1_{\text {stat }}^{\prime} \pm 1.0_{\text {sys }}^{\prime}$ \\
\hline$\sigma_{\mathrm{src}}$ & $6.2^{\prime}$ & $\pm 1.2_{\text {stat }}^{\prime} \pm 0.9_{\text {sys }}^{\prime}$ \\
\hline
\end{tabular}

(b) Tight cuts: $\theta<0.12^{\circ}, \bar{w}<1.1, n_{\text {tel }} \geq 3$

\begin{tabular}{lccccc}
\hline \hline $\begin{array}{l}\text { Background } \\
\text { model }\end{array}$ & $s$ & $b$ & $\alpha$ & $s-\alpha b$ & $S$ \\
\hline \multicolumn{5}{c}{-1999 to 2001 Dataset $\$ 1(120.5 \mathrm{~h})-$} \\
Template & 529 & 2432 & 0.168 & 123 & $\mathbf{+ 5 . 3}$ \\
Displaced & 529 & 6982 & 0.059 & 119 & $\mathbf{+ 5 . 4}$ \\
& -2002 & Dataset $\S 2$ & $(157.8 \mathrm{~h})$ & - & \\
Template & 716 & 3494 & 0.168 & 129 & $\mathbf{+ 4 . 8}$ \\
Displaced & 716 & 8510 & 0.070 & 125 & $\mathbf{+ 4 . 8}$ \\
\multicolumn{7}{c}{ Template } & 1245 & 5926 & 0.168 & 252 & $\mathbf{+ 7 . 1}$ \\
Displaced & 1245 & 15492 & 0.065 & 243 & $\mathbf{+ 7 . 1}$ \\
\hline
\end{tabular}

(c) Spectral Cuts: Tight Cuts + core $\leq 200 \mathrm{~m}$

Energy estimation method: See Hofmann et al. (2000b)

\begin{tabular}{|c|c|c|c|c|c|}
\hline $\begin{array}{l}\text { Background } \\
\text { model }\end{array}$ & $s$ & $b$ & $\alpha$ & $s-\alpha b$ & $S$ \\
\hline \multicolumn{6}{|c|}{-1999 to 2001 Dataset $\$ 1(120.5 \mathrm{~h})-$} \\
\hline Displaced & 421 & 2120 & 0.143 & 118 & +6.0 \\
\hline \multicolumn{6}{|c|}{ — 2002 Dataset $\S 2(157.8 \mathrm{~h})-$} \\
\hline Displaced & 552 & 2999 & 0.143 & 124 & +5.3 \\
\hline \multicolumn{6}{|c|}{ — All Data $(278.3 \mathrm{~h})$ — } \\
\hline Displaced & 973 & 5119 & 0.143 & 242 & +7.9 \\
\hline
\end{tabular}

differences in $\mathrm{CoG}$ obtained from all three available algorithms " 1 ", " 2 " and "3". Simulations and empirical results from other point sources of the HEGRA IACT-System archive have shown that consistent sensitivities from all algorithms are expected for a minimum number of images per event $n_{\text {tel }} \geq 3$. In fact the signal excess significance from all data using each algorithm are consistent to within $1 \sigma$, and the CoGs and source extensions agree to within a $2 \sigma$ level. Taking the final CoG and source extension from algorithm " 3 ", the respective systematic errors were taken using results from the other algorithms. The excess significance from combined data exceeds 
Table 2. Numerical summary for TeV J2032+4130 continued.. d) differential fluxes and events after tight spectral cuts; e) fitted power law; f) integral flux.

\begin{tabular}{|c|c|c|c|c|c|}
\hline & \multicolumn{4}{|c|}{ (d) Spectral Cuts: Differential points } & \multirow[b]{2}{*}{$S^{b}$} \\
\hline $\begin{array}{l}\text { Energy } \\
E(\mathrm{TeV})\end{array}$ & Flux $(E)^{a}$ & $\begin{array}{c}\text { Flux }^{a} \\
\text { Error }(E)\end{array}$ & $s$ & $b$ & \\
\hline \multicolumn{6}{|c|}{-1999 to 2001 Dataset $\$ 1(120.5 \mathrm{~h})-$} \\
\hline 1.05 & 1.70 & 1.24 & 130 & 678 & +3.0 \\
\hline 1.82 & 1.94 & 0.91 & 107 & 531 & +3.1 \\
\hline 3.16 & 0.37 & 0.27 & 57 & 323 & +1.4 \\
\hline 5.50 & 0.30 & 0.11 & 33 & 117 & +3.2 \\
\hline 9.55 & 0.09 & 0.04 & 13 & 34 & +2.8 \\
\hline \multicolumn{6}{|c|}{ — 2002 Dataset $\S 2(157.8 \mathrm{~h})-$} \\
\hline 1.05 & 19.90 & 16.57 & 153 & 885 & +2.1 \\
\hline 1.82 & 1.85 & 1.56 & 152 & 913 & +1.7 \\
\hline 3.16 & 0.85 & 0.28 & 107 & 513 & +3.4 \\
\hline 5.50 & 0.28 & 0.10 & 44 & 174 & +3.2 \\
\hline 9.55 & 0.07 & 0.04 & 17 & 51 & +2.8 \\
\hline \multicolumn{6}{|c|}{ — All Data $(278.3 \mathrm{~h})$ - } \\
\hline 1.05 & 11.98 & 10.80 & 283 & 1563 & +3.6 \\
\hline 1.82 & 1.89 & 0.97 & 259 & 1444 & +3.3 \\
\hline 3.16 & 0.64 & 0.20 & 164 & 836 & +3.6 \\
\hline 5.50 & 0.29 & 0.07 & 77 & 291 & +4.5 \\
\hline 9.55 & 0.08 & 0.03 & 30 & 85 & +3.9 \\
\hline
\end{tabular}

(e) Fitted Spectrum: Pure Power-Law

\begin{tabular}{ccc}
\hline \hline $\mathrm{d} N / \mathrm{d} E$ & $=$ & $N(E / 1 \mathrm{TeV})^{-\gamma} \mathrm{ph} \mathrm{cm}^{-2} \mathrm{~s}^{-1} \mathrm{TeV}^{-1}$ \\
-1999 to 2001 Dataset $\S 1(120.5 \mathrm{~h})-$ \\
$N \quad=$ & $4.1\left( \pm 2.1_{\text {stat }} \pm 1.3_{\text {sys }}\right) \times 10^{-13}$ \\
$\gamma$ & $=$ & $1.7\left( \pm 0.3_{\text {stat }} \pm 0.3_{\text {sys }}\right)$ \\
& -2002 Dataset $\S 2(157.8 \mathrm{~h})-$ \\
$N$ & $=$ & $9.3\left( \pm 2.9_{\text {stat }} \pm 1.4_{\text {sys }}\right) \times 10^{-13}$ \\
$\gamma$ & $=$ & $2.1\left( \pm 0.2_{\text {stat }} \pm 0.3_{\text {sys }}\right)$ \\
& - All Data $(278.3 \mathrm{~h})-$ \\
$N$ & $=$ & $6.2\left( \pm 1.5_{\text {stat }} \pm 1.3_{\text {sys }}\right) \times 10^{-13}$ \\
$\gamma$ & $=$ & $1.9\left( \pm 0.1_{\text {stat }} \pm 0.3_{\text {sys }}\right)$ \\
\hline
\end{tabular}

\begin{tabular}{c} 
(f) Integral Flux ${ }^{a}(\mathbf{E}>\mathbf{1 ~ T e V})$ \\
\hline \hline-1999 to 2001 Dataset $\$ 1(120.5 \mathrm{~h})-$ \\
$F(E>1 \mathrm{TeV})=5.86\left( \pm 3.91_{\text {stat }}\right)$ \\
-2002 Dataset $\$ 2(157.8 \mathrm{~h})-$ \\
$F(E>1 \mathrm{TeV})=8.45\left( \pm 3.05_{\text {stat }}\right)$ \\
- All Data $(278.3 \mathrm{~h})-$ \\
$F(E>1 \mathrm{TeV})=6.89\left( \pm 1.83_{\text {stat }}\right)$ \\
\hline${ }^{a}$ Flux and Errors in units $\times 10^{-13} \mathrm{ph} \mathrm{cm}^{-2} \mathrm{~s}^{-1}$.
\end{tabular}

$7 \sigma(+7.1 \sigma)$, and the source appears extended at the $>4 \sigma$ level when, conservatively, subtracting the systematic error in extension. The source is termed $\mathrm{TeV} \mathrm{J} 2032+4130$ based on its CoG. The post-trial significance is $\sim 6.1 \sigma$ if one assumes the trial factors (1100) accrued from the discovery dataset $\$ 1$ (Aharonian et al. 2002). The radial source extension is shown in Fig. 1 and compared against a number of possible source morphologies. We tested three different types of intrinsic source morphology:

- Disc: the source emits at a constant brightness out to a radius $0.13^{\circ}$ (with zero brightness outside). This profile resembles that of a disc.

- Volume: the source emits at all positions inside a sphere of radius $0.13^{\circ}$.

- Surface: the source emits at all positions within a radial band between $0.117^{\circ}$ and $0.13^{\circ}$.

In each case the volume-integrated radial brightness is calculated after convolution with the instrument PSF, and compared with the measured radial profile of $\mathrm{TeV} \mathrm{J} 2032+4130$. In all cases similar reduced- $\chi^{2} \sim 1.0$ were obtained, thereby not permitting discrimination between these source morphologies.

Figure 2 depicts the 2D skymap of excess significance over the FoV, using the template model as a CR background estimate. TeV J2032+4130 is clearly positioned at the edge of

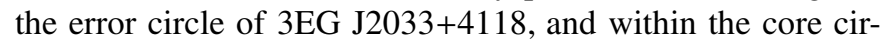
cle of the extremely dense OB stellar association Cygnus OB2 (Knödlseder 2000).

The energy spectrum determination followed the method described in Aharonian et al. (1999) using tight cuts (on $\theta$ and $\bar{w})$ plus an additional cut on the reconstructed air-shower core distance of the event core $<200 \mathrm{~m}$. Reconstruction of event energies employed the more advanced method of Hofmann et al. (2000b) which makes use of the height of shower maximum to improve the core distance determination, and hence improve energy resolution to $<15 \%$. Both datasets $\S 1$ and $\S 2$ yielded consistent power-law fits with a hard photon index. For all data, a pure power law explains well the energy spectrum, showing no indication for a cut-off when fitting also a combined power law + exponential cutoff term $\exp \left(-E / E_{\mathrm{c}}\right)$. We estimated, nevertheless, lower limits ( $99 \%$ c.l.) to the cut-off energy for a range of (fixed) power law indices. Cut-off lower limits of $E_{\mathrm{c}} \sim 3.6,4.2$ and $4.6 \mathrm{TeV}$ result when fixing the power index at values $\gamma=1.7,1.9$, and 2.2 , respectively.

In estimating all flux values we have assumed a Gaussian source profile according to the estimated source size since the non-pointlike nature of the source is confirmed. Note that previously published integral fluxes for combined data (Rowell et al. 2003; Horns et al. 2004) assumed a pointlike source. It is also apparent that the event rate for dataset $\S 2$ is $\sim 80 \%$ that of dataset $\S 1$, and that the integral fluxes (for $E>1 \mathrm{TeV}$ ) derived differ by about $40 \%$. However the statistical errors on the integral flux, which are dominated by contributions near $1 \mathrm{TeV}$, suggests this difference is not significant $(\sim 1 \sigma)$.

We conclude therefore that $\mathrm{TeV}$ J2032+4130 exhibited a constant flux from 1999 to 2002 . Note that the integral (and differential) fluxes are corrected for changes in the IACT-System response over time (see Pühlhofer et al. 2003, for details on the system performance over time). In this case, corrections up to the individual run level according to the $\mathrm{CR}$ background rate 

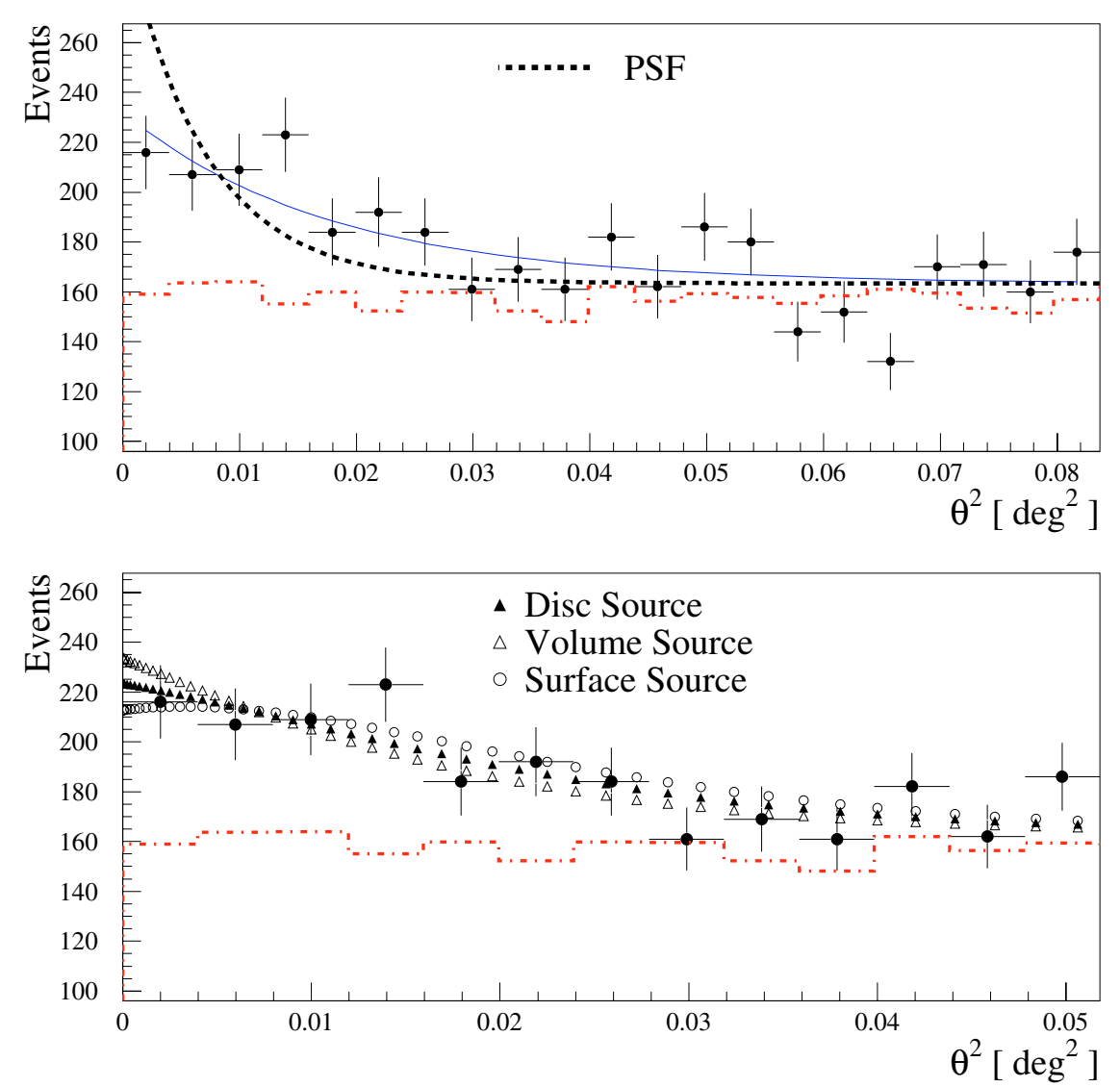

Fig. 1. (Top) Events (solid points with error bars) vs. distance from the CoG squared $\theta^{2}$ compared with a background estimate from the template model (dashed-dotted line). A convolved radial Gaussian fit $F=P e d+P_{2} \exp \left(-\theta^{2} /\left(P_{1}^{2}+\sigma_{\mathrm{pt}}^{2}\right)\right)$ is indicated by the solid line with $P_{1}=\sigma_{\text {src }}=$ $0.104^{\circ} \pm 0.020^{\circ}$ the intrinsic source size. The PSF width is $\sigma_{\mathrm{pt}}=0.070^{\circ}$ (dashed). The pedestal Ped is fitted separately. (Bottom) Comparisons with various intrinsic source morphologies convolved with the PSF (see text).

(a reliable, relative measure of the detector+atmospheric transmission) have been applied.

We also include in Table 3, 99\% upper limits for a number of other interesting sources in the FoV. These include the two GeV sources, their possible associated X-ray counterparts (as indicated by Roberts et al. 2001), and also Cygnus X-3.

\section{Discussion and conclusion}

Possible origins of TeV J2032+4130 have been considered in Aharonian et al. (2002), Butt et al. (2003), Mukherjee et al. (2003) and Bednarek (2003). One interpretation involves association with the stellar winds of member stars in Cygnus OB2, individually or collectively, which provide conditions conducive to strong and stable shock formation for particle acceleration. Another scenario involves particle acceleration at a termination shock, which is expected at the boundary where a relativistic jet meets the interstellar medium. TeV J2032+4130 in fact aligns well within the northern error cone of the bi-lobal jet of Cygnus X-3 discussed by Martí et al. (2000, 2001). The existence of $\mathrm{TeV}$ emission clearly suggests that particles are accelerated to at least multi-TeV energies. Taken at face value the different flux levels (a maximum factor $\sim 20$ difference) claimed by the Crimean (1.7 Crab $E>1 \mathrm{TeV}$ ), HEGRA (0.05 Crab $E>1 \mathrm{TeV})$ and Whipple $(0.12 \mathrm{Crab} E>0.6 \mathrm{TeV})$ groups
Table 3. Summary of point-like flux upper limits for other sources in the FoV. Positions for the various X-ray sources are taken from Roberts et al. (2001). An energy threshold of $E_{\text {th }}>0.7 \mathrm{TeV}$ is estimated based on the average zenith angle $\bar{z}$ of observations: $E_{\mathrm{th}}=$ $0.5 \cos (\bar{z})^{-2.5}$. The template background model been used $(\alpha=0.168)$, with $s, b$ and $S$ defined as in Table 1.

Other Sources: (tight cuts) $\theta<0.12^{\circ}, \bar{w}<1.1, n_{\text {tel }} \geq 3$

\begin{tabular}{lccccc}
\hline \hline Source & $s$ & $b$ & $s-\alpha b$ & $S$ & ${ }^{a} \phi^{99 \%}$ \\
\hline GeV J2026+4124 & 541 & 3429 & -35 & $\mathbf{- 1 . 3}$ & $\mathbf{0 . 2 9}$ \\
AX J2027.6+4116 & 771 & 4497 & 16 & $\mathbf{+ 0 . 6}$ & $\mathbf{0 . 4 2}$ \\
GeV J2035+4214 & 784 & 4457 & 35 & $\mathbf{+ 1 . 2}$ & $\mathbf{0 . 4 5}$ \\
AX J2036.0+4218(Src1) & 620 & 4005 & -53 & $-\mathbf{1 . 8}$ & $\mathbf{0 . 2 2}$ \\
AX J2035.4+4222(Src2) & 663 & 4083 & -23 & $\mathbf{- 0 . 7}$ & $\mathbf{0 . 2 7}$ \\
AX J2035.9+4229(Src3) & 584 & 3626 & -25 & $\mathbf{- 0 . 9}$ & $\mathbf{0 . 2 8}$ \\
Cygnus X-3 & 857 & 5613 & -86 & $\mathbf{- 2 . 6}$ & $\mathbf{0 . 1 7}$ \\
\hline
\end{tabular}

${ }^{a} \phi_{\mathrm{ph}}^{99 \%}=99 \%$ upper limit $E_{\mathrm{th}}>0.7 \mathrm{TeV}\left[\times 10^{-12} \mathrm{ph} \mathrm{cm}^{-2} \mathrm{~s}^{-1}\right]$.

over a period of a decade, would suggest episodic emission from TeV J2032+4130. Explaining also the extended nature of TeV J2032+4130 as seen by HEGRA requires consideration of issues such as the particle acceleration site and its distance to that of the $\mathrm{TeV} \gamma$-ray emission, particle diffusion and source 


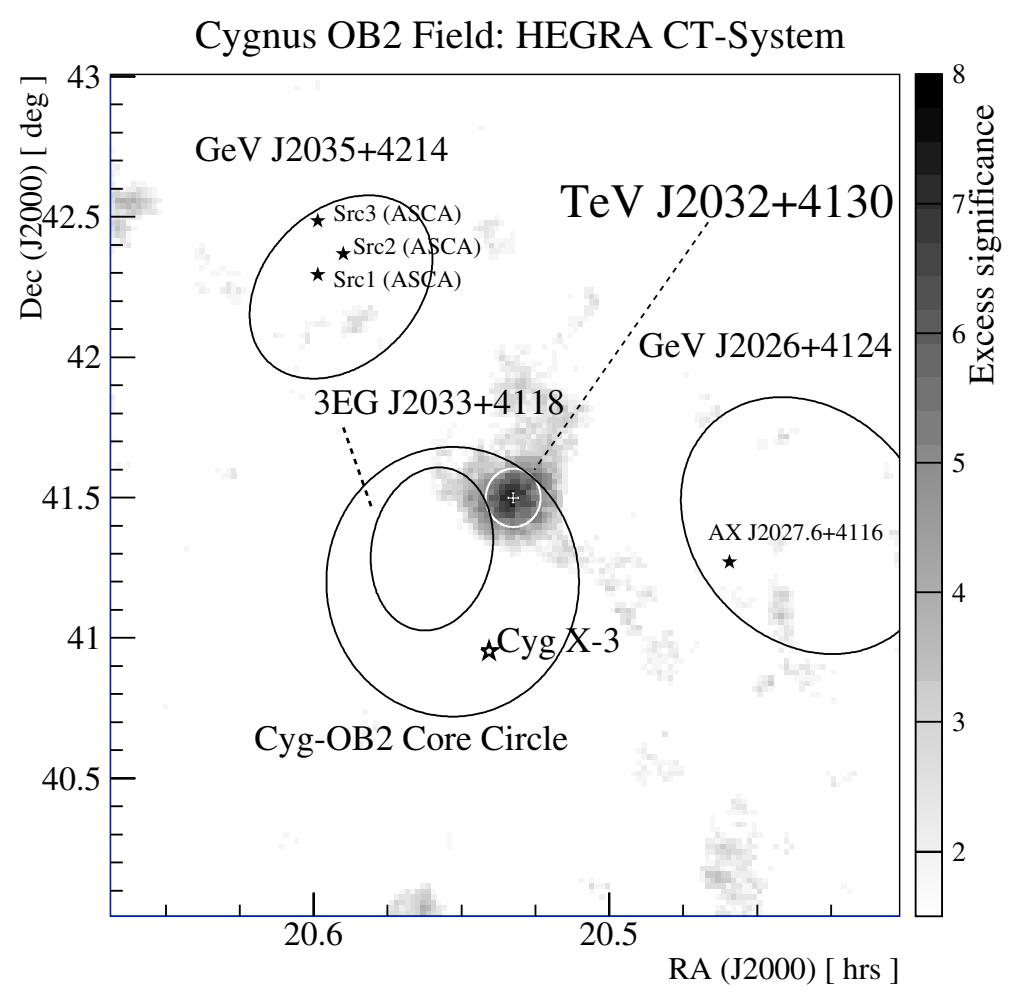

Fig. 2. Skymap of correlated event excess significance $(\sigma)$ from all HEGRA IACT-System data $\left(3.0^{\circ} \times 3.0^{\circ}\right.$ FoV $)$ centred on TeV J2032+4130. Nearby objects are indicated (EGRET sources with $95 \%$ contours). The TeV source centre of gravity (CoG) with statistical errors, and intrinsic size (std. dev. of a $2 \mathrm{D}$ Gaussian, $\sigma_{\text {src }}$ ) are indicated by the white cross and white circle, respectively.

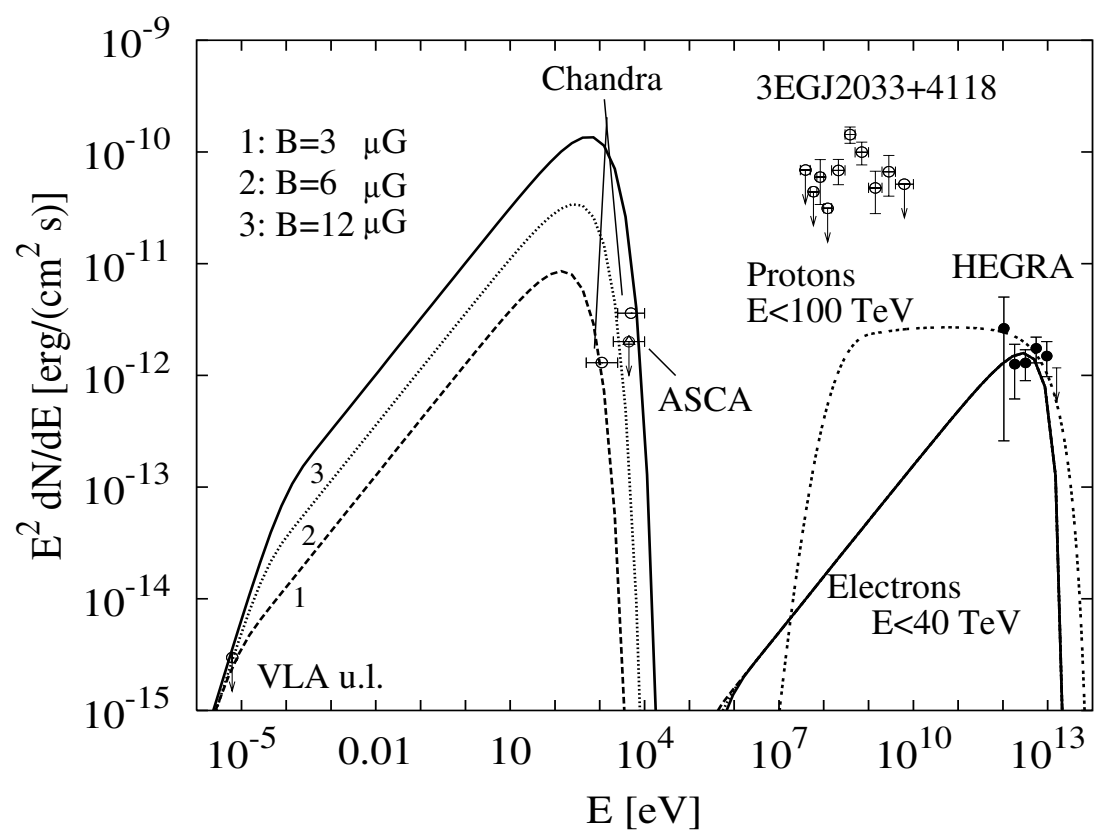

Fig. 3. Spectrum of TeV J2032+4130 (this work - HEGRA) compared with purely hadronic (Protons $E<100 \mathrm{TeV}$ ) and leptonic (Electrons $E<40 \mathrm{TeV}$ ) models. Upper limits, constraining the synchrotron emission (leptonic models), are from the VLA and Chandra (Butt et al. 2003) and ASCA (Aharonian et al. 2002). In the model a minimum electron energy $\gamma_{\min } \sim 10^{4}$ is chosen to meet the VLA upper limit. EGRET data points are from the 3rd EGRET catalogue (Hartman et al. 1999).

age. Such issues would also not in general rule out an extended and episodic source. Moreover the TeV J2032+4130 emission at any given time may be a superposition of more than one component (e.g. variable compact in addition to weak, steady, extended emission).
For illustrative purposes we have matched the spectral energy distribution of TeV J2032+4130 with simplistic leptonic and hadronic models (Fig. 3). We assume that the TeV emission arises from a single pure population of either non-thermal hadronic or electronic parent particles. We do not consider here 
the conditions under which particles are accelerated or how they lose energy. Under the hadronic scenario the $\pi^{\circ}$-decay prediction matches well the $\mathrm{TeV}$ flux using a parent proton power law spectrum of index -2.0 with a sharp limit at $100 \mathrm{TeV}$. The neighbouring EGRET source 3EG J2033+4118 (possibly not related to $\mathrm{TeV} \mathbf{J} 2032+4130$ ) should be considered here as upper limits on the potential GeV flux of TeV J2032+4130. Associated synchrotron X-ray emission would also be expected from tertiary electrons $\left(\pi^{ \pm} \ldots \rightarrow \mu^{ \pm} \ldots \rightarrow e^{ \pm}\right)$, not modeled at present, which represent an absolute lower limit on any synchrotron emission visible assuming a pure electronic scenario. TeV data are matched well by an inverse-Compton spectrum (from electrons up-scattering the cosmic microwave background) arising from an electron spectrum with power law index $\sim-2.0$ and a sharp limit at $40 \mathrm{TeV}$. The predicted synchrotron emission then follows as a function of local magnetic field, constrained by the available upper limits at radio and $\mathrm{X}$-ray energies. The most conservative synchrotron prediction arises for $B_{0}=3 \mu \mathrm{G}$, the lowest such B-field expected in the Galactic disk. In fact, much higher B-fields $\left(B_{0}>10 \mu \mathrm{G}\right)$ are generally expected in such regions containing young/massive stars with high mass losses and colliding winds (e.g. Eichler et al. 1993). X-ray results from ASCA (Aharonian et al. 2002) provide constraining upper limits, as do results from Chandra (Butt et al. 2003). Deeper observations by XMM and Chandra will no doubt provide further constraints on the leptonic component. Future $\gamma$-ray observations by H.E.S.S., VERITAS and also MAGIC-II will be vital in better determining the spectrum over the $\sim 50 \mathrm{GeV}$ to $>10 \mathrm{TeV}$ regime. Knowledge of the spectral behaviour for $E>10 \mathrm{TeV}$ will convey important information on the maximum particle energies and their type. Such information can come from low elevation observations by H.E.S.S. for which very large collecting areas are achieved at $\mathrm{TeV}$ energies. Energy-resolved morphology studies can also be performed, allowing conclusions on the diffusion properties of the accelerated particles. Overall, TeV J2032+4130 is the only $\mathrm{TeV}$ source so far without an obvious multiwavelength counterpart, and is likely the first-discovered galactic $\mathrm{TeV}$ source which is extended in nature.

The serendipitous detection of such a weak ( $\sim 0.05 \mathrm{Crab})$, marginally extended source with a hard spectrum over a long observation time illustrates the power of the stereoscopic technique as was employed by the HEGRA IACT-System. With a sensitivity at least a factor of 5 better, the next generation instruments will find such sources detectable in under $10 \mathrm{~h}$, or even less for sources with steeper energy spectra.

Finally, we also obtained upper limits (for a steady $\mathrm{TeV}$ flux) from a number of other source positions in the FoV (Fig. 2, Table 3), including the famous microquasar Cygnus X-3 for which an upper limit $(E>0.7 \mathrm{TeV}) 1.7 \times$ $10^{-13} \mathrm{ph} \mathrm{cm}^{-2} \mathrm{~s}^{-1}$ for steady emission is set.
Acknowledgements. The support of the German Ministry for Research and Technology BMBF and of the Spanish Research Council CICYT is gratefully acknowledged. We thank the Instituto de Astrofísica de Canarias for the use of the site and for supplying excellent working conditions at La Palma. We gratefully acknowledge the technical support staff of the Heidelberg, Kiel, Munich and Yerevan Institutes. GPR acknowledges receipt of a von Humboldt fellowship.

\section{References}

Aharonian, F. A., Akhperjanian, A. G, Barrio, J. A., et al. 1999, A\&A, 349,11

Aharonian, F. A., Akhperjanian, A. G., Barrio, J. A., et al. 2000a, ApJ, 539,317

Aharonian, F. A, Akhperjanian, A. G., Barrio, J. A., et al. 2000b, A\&A, 361, 1073

Aharonian, F. A., Akhperjanian, A., Beilicke, M., et al. 2002, A\&A, 393, L37

Aharonian, F. A., Akhperjanian, A., Beilicke, M., et al. 2004, ApJ, in press [arXiv: astro-ph/0407118]

Bednarek, W. 2003, MNRAS, 345, 847

Butt, Y., Benaglia, P., Combi, J. A., et al. 2003, ApJ, 597, 494

Eichler, D., \& Usov, V. 1993, ApJ, 402, 271

Hartman, R. C., Bertsch, D. L., Bloom, S. D., et al. 1999, ApJS, 123 , 79

Hofmann, W., Jung, I., Konopelko, A., et al. 1999, Astropart. Phys., 10,275

Hofmann, W., Akhperjanian, A. G, Barrio, J. A., et al. 2000a, A\&A, 361,1073

Hofmann, W., Lampeitl, H., Konopelko, A., et al. 2000b, Astropart. Phys., 12, 135

Horns, D., Rowell, G. P., et al. 2004, New AR, 48, 489

Knödlseder, J. 2000, A\&A, 360, 539

Krawczynski, H. 1995, Diplom Thesis, University of Hamburg, Hamburg

http://www-hegra.desy.de/publications.html

Lang, M. J., Carter-Lewis, D. A., Fegan, D. J., et al. 2004, A\&A, 423, 415

Li, T. P., \& Ma, Y. Q. 1983, ApJ, 272, 317

Merck, M. 1993, Ph.D. Dissertation, Ludwig-Maximilians University, Munich

Martí, J., Paredes, J. M., Peracaula, M., et al. 2000, ApJ, 545, 939

Martí, J., Paredes, J. M., Peracaula, M., et al. 2001, A\&A, 375, 476

Mukherjee, R., Halpern, J. P., Gotthelf, E. V., et al. 2003, ApJ, 589, 487

Neshpor, Y. I., Kalekin, O. R., Stepanian, A. A., et al. 1995, Proc. 24th ICRC (Rome), 2, 1385

Pühlhofer, G., Bolz, O., Götting, N., et al. 2003, Astropart. Phys., 20, 267

Roberts, S. E., Romani, R. W., \& Kawai, N. 2001, ApJS, 133, 451

Rowell, G. P. 2003, A\&A, 410, 389

Rowell, G. P., et al. 2003, Proc. 28th ICRC Tsukuba, OG 2.2, 2345 [arXiv: astro-ph/0307334] 